\title{
DISMINUCIÓN DE LA INCIDENCIA DE ÚlCERAS POR PRESIÓN EN CUIDADOS INTENSIVOS: OBJETIVO DE UN PROGRAMA DE MEJORA ASISTENCIAL.
}

\section{DECREASED INCIDENCE OF PRESSURE ULCERS IN INTENSIVE CARE: A PROGRAM GOAL OF IMPROVING CARE.}

Romero de San Pío, Emilia; Romero de San Pío, Maria Jesús; Valdés Fernández, Maria Teresa; Alonso Rodríguez, Aránzazu; Secades Gamazo, María Lara; Santa Cruz Rodríguez, María Isabel; Rodríguez Villanueva, Lara María; Rodríguez Rodríguez, Ana Isabel; Patallo Fernández, María Teresa; Linares Gutiérrez, Begoña; González García, Isabel; García Rato, Jezabel; García García, Diana; Clavero Ballester, Natividad; Álvarez Prado, María Candela; Alonso Álvarez, Teresa; Ana Manuela Fernández Fernández; Ana Joaquina Martínez Fernández.

\section{E-mail contacto: emiliaromero@telefonica.net}

UCI-1. Hospital Universitario Central de Asturias. 


\section{RESUMEN}

\section{TÍTULO.}

Disminución de la incidencia de úlceras por presión en Cuidados Intensivos: objetivo de un programa de mejora asistencial.

\section{INTRODUCCIÓN.}

En sanidad, la aparición de úlceras por presión (upp), es considerado un fracaso asistencial y un gran reto al que se enfrenta el profesional de Enfermería. En cuidados intensivos, objetivamos una serie de factores que aumentan el riesgo de aparición y desarrollo de upp. Como enfermeros, tomamos conciencia de nuestro papel en la prevención y tratamiento de las upp en nuestra unidad de cuidados intensivos (UCI-1, Hospital Universitario Central de Asturias) y emprendimos medidas para disminuirlas y mejorar la calidad asistencial proporcionada.

\section{OBJETIVOS.}

Aumentar la calidad asistencial

Disminuir las tasas de úlceras por presión

\section{MATERIAL Y MÉTODO.}

Estudio descriptivo, prospectivo. Muestra, N: 1265 enfermos. Realizándose seguimiento diario de enfermos a través de hoja de valoración específica. Los enfermos valorados presentaban riesgo medio- alto de padecer upp por escala Braden y riesgo bajo por dicha escala pero con condiciones particulares de riesgo objetivables. Se introducen medidas preventivas consensuadas por el equipo investigador (descritas en el trabajo de investigación).

\section{RESULTADOS.}

Bajada significativa de las tasas de incidencia a los cuatro meses de introducir medidas estandarizadas de prevención y mejora asistencial.

\begin{tabular}{|l|c|c|c|c|}
\hline $\begin{array}{c}\text { Tasas medias } \\
\text { (porcentajes) }\end{array}$ & $\begin{array}{c}\text { Datos pre- } \\
\text { estudio }\end{array}$ & $\begin{array}{c}\text { Datos estudio } \\
\mathbf{( 2 0 0 9 )}\end{array}$ & $\begin{array}{c}\text { Datos estudio } \\
\mathbf{( 2 0 1 0 )}\end{array}$ & $\begin{array}{c}\text { Datos estudio } \\
\text { (globales) }\end{array}$ \\
\hline Prevalencia & 45,98 & 20,22 & 14,30 & 17,76 \\
\hline Incidencia & 45,18 & 16,52 & 9,22 & 12,21 \\
\hline
\end{tabular}

Tabla 2: comparativa de tasas.

\section{CONCLUSIONES.}

La concienciación y unificación de criterios preventivos y de tratamiento resultó ser decisiva en la bajada significativa de la incidencia de upp y en el logro de aumento de la calidad asistencial real percibida por todo el equipo interdisciplinar.

PALABRAS CLAVE.

Calidad asistencial, cuidados intensivos, úlceras por presión, Enfermería. 


\section{ABSTRAT}

\section{TITLE:}

Decreased incidence of pressure ulcers in intensive care: a program goal of improving care.

\section{INTRODUCTION.}

In health, the occurrence of pressure ulcers (PU) is considered a failure of care and major challenge facing the nursing professional. In intensive care, objectify a series of factors that increase the risk of occurrence and development of PU. As nurses, we realize our role in the prevention and treatment of pressure ulcers in our intensive care unit (ICU-1, Hospital Universitario Central de Asturias) and we took measures to reduce and improve quality of care provided.

\section{OBJECTIVES.}

To augment quality of care

To diminish rates of pressure ulcers

\section{MATERIALS AND METHODS.}

Prospective descriptive study. Sample, $\mathrm{n}=1265$ patients. Performing daily monitoring of patients through specific assessment sheet. The patients had rated medium-high risk of developing upp by Braden scale and low risk for this scale but with risk conditions to measure. Agreed preventive measures are introduced by the research team (described in the research).

\section{RESULTS.}

Llower incidence rates for four months to introduce standardized measures of prevention and improved care.

\begin{tabular}{|l|c|c|c|c|}
\hline $\begin{array}{c}\text { Average rates } \\
\text { (percentages) }\end{array}$ & Data pre-study & $\begin{array}{c}\text { Data study } \\
\mathbf{( 2 0 0 9 )}\end{array}$ & $\begin{array}{c}\text { Data study } \\
\mathbf{( 2 0 1 0 )}\end{array}$ & $\begin{array}{c}\text { Data study } \\
\text { (globales) }\end{array}$ \\
\hline Prevalence & 45,98 & 20,22 & 14,30 & 17,76 \\
\hline Incidence & 45,18 & 16,52 & 9,22 & 12,21 \\
\hline
\end{tabular}

Table 2: comparison of rates.

\section{CONCLUSIONS.}

The unification of awareness and prevention and treatment criteria proved to be instrumental in the significant decline in the incidence of PU and the achievement of increased quality of care received by all real interdisciplinary team.

\section{KEYWORDS.}

quality care, intensive care, pressure sores, Nursing. 


\section{INTRODUCCIÓN.}

En la sanidad actual, con sus características especiales, desarrollo y prestaciones, la aparición de úlceras por presión (upp) en pacientes ingresados, es considerada un fracaso asistencial y uno de los principales retos a los que se debe de enfrentar el profesional de Enfermería en su trabajo diario con los pacientes a su cargo. Dentro de las unidades de cuidados intensivos, el reto, si cabe, es mayor ya que nos enfrentamos a una serie de factores generales y particulares que aumentan significativamente el riesgo de aparición y desarrollo de upp:

$>$ Pacientes sometidos a sedación y relajación.

$>$ Inmovilidad física.

$>$ Deterioro sensorial.

> Gravedad del proceso patológico.

> Polimedicaciones que pueden ocasionar deterioro circulatorio.

Múltiples trabajos científicos correlacionan directamente el aumento de la morbi-mortalidad y la estancia media hospitalaria a la aparición de una úlcera por presión y a su estadio de gravedad. Debido a todo ello, como profesionales de Enfermería, tomamos conciencia de nuestro papel protagonista en la prevención y tratamiento de las upp y emprendimos en nuestra unidad de cuidados intensivos de referencia (UCI-1 del Hospital Universitario Central de Asturias) una serie de medidas para disminuir la incidencia y prevalencia de upp que sufríamos y mejorar así la calidad asistencial proporcionada a nuestros enfermos.

Nuestros objetivos tanto a nivel general como objetivos desde el punto de vista específico, fueron los siguientes:

\section{Objetivo general:}

- Aumentar la calidad asistencial.

- Disminuir las tasas de úlceras por presión. 


\section{Objetivos específicos:}

- Instaurar, a la luz de los conocimientos actuales, un nuevo modelo teórico de actuación en la prevención y tratamiento de las upp en cuidados intensivos (UCI).

- Adecuar los modelos preventivos disponibles a la realidad de nuestro medio.

- Disminuir las tasas de prevalencia e incidencia de upp.

\section{MATERIAL Y MÉTODO.}

Estudio descriptivo, prospectivo realizado en la UCI-1 del Hospital Universitario Central de Asturias (Oviedo).

Muestra, n: 1265 enfermos, con una media de ingresos de 58,40+/-7,5 enfermos/mes.

Se realizó un seguimiento diario de los enfermos ingresados en UCI a través de una hoja de valoración diaria que se encontraba introducida en la historia clínica de cada paciente y que cumplimentaban cada uno de los profesionales de Enfermería responsables de cada paciente (Esquema 1).Los enfermos que fueron valorados presentaban un riesgo medio- alto de padecer ùlceras por presión (upp) por escala de valoración: Braden y riesgo bajo por dicha escala pero con ciertas condiciones intrínsecas y extrínsecas de riesgo objetivables.

Se contabilizaron las upp aparecidas, realizándose un estudio mensual de las tasas de prevalencia e incidencia, durante 22 meses, desde abril de 2009 a enero de 2011, ambos inclusive. Criterio único de exclusión: estancia en UCI inferior a 24 horas.

Se formó un equipo de 18 profesionales enfermeros que directamente se encargaron del estudio y seguimiento de las úlceras por presión durante todos los meses descritos. Dicho grupo emprendió una serie de actividades previas que se consensuaron como básicas para lograr el objetivo principal de disminución de las tasas de upp y mejora asistencial. 
Dichas actividades fueron las siguientes:

- Revisión y actualización del protocolo de prevención de upp.

- Realización de un protocolo actualizado de correcta higiene corporal del paciente crítico.

- Unificación de tratamientos de las úlceras según gravedad y estadío.

- Instauración en el almacén de nuestra UCI de un lugar específico donde se colocaron los distintos dispositivos de tratamiento de upp con paneles informativos y algoritmo terapéutico.

- Cambio de la escala de valoración del riesgo de padecer upp. Desestimación de la escala previa utilizada en el servicio: Norton por la de Braden que ha demostrado su mayor sensibilidad, especificidad y mayor valor predictivo en la discriminación de los pacientes con mayor riesgo de padecer upp.

- Incorporamos una metodología de estudio y seguimiento lineal de las frecuencias absolutas de upp.

- Reuniones periódicas del equipo de seguimiento y control de las upp.

A partir de abril del 2009 se fueron introduciendo las medidas preventivas y de tratamiento consensuadas. Se obtuvo:

- Concienciación general sobre un grave problema asistencial que sufríamos.

- Unificación de criterios preventivos y de tratamiento de las upp.

Se presenta en el siguiente esquema el documento de seguimiento del programa: 


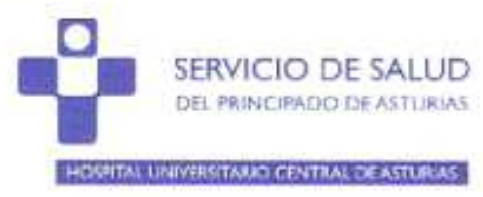

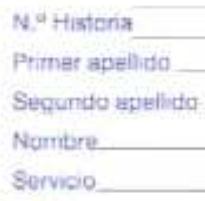

Fectla de ingreso
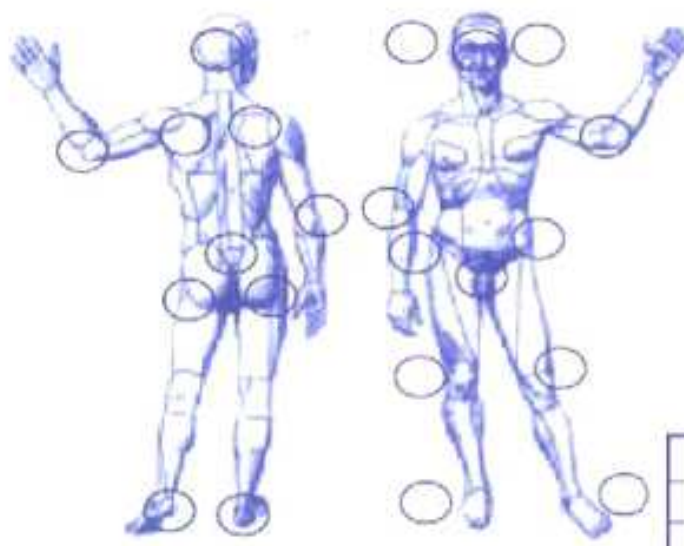

Procedencia UPP:

Hospitalaria $1 \square 2 \square 3 \square 4$

Extrahospitalaria 1|| $2|\quad 3 \square| 4 \square$

\begin{tabular}{|c|c|c|c|l|}
\cline { 2 - 5 } \multicolumn{1}{c|}{} & Fecha & Medida & Estadio & Tunelización \\
\hline UPP 1 & & $\mathrm{x}$ & & \\
\hline UPP 2 & & $\mathrm{x}$ & & \\
\hline UPP 3 & & $\mathrm{x}$ & & \\
\hline UPP 4 & & $\mathrm{x}$ & & \\
\hline
\end{tabular}

$\begin{array}{llll}\text { DIA DIA } & \text { DIA } & \text { DIA } & \text { DIA }\end{array}$

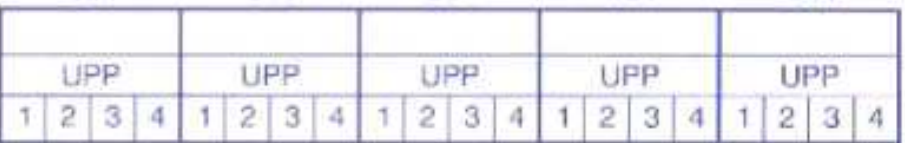

UPP DESCRIPCION DEL LECHO DE LA HERIDA:

Tejido necrótico o esfacelado

Tejido de granulación

Epitelización

Signos infección

Próxima cura (sertalar con una $X$ )

NIVEL DE EXUDADO:

N: Ninguno / E: Escaso / M: Moderado / A: Alto

ASPECTO DEL EXUDADO:

S: Seroso / H: Hematico / P: Purulento

ESTADO DE LA PIEL PERILESIONAL:

1: integra / L: Lacerada / M: Macerada / C: Celulitis

TRATAMIENTO LOCAL APLICADO:

Apósito Hidrocoloide

Apósito Poliuretano (Hidrocelulat o Hicropolimetico

Apósito Hidrostático

Alginato (Hidrocoloido fibra)

Carbón activado

Apósito plata

Hidrogel

Acicios grasos hiperoxigenados

Desbridamiento enzimatico (Colagenasa)

Desbridarniento M: Mecánico/ A: Autolítico

Cultivo

Otros

FIRMA ENFERMERA / 0
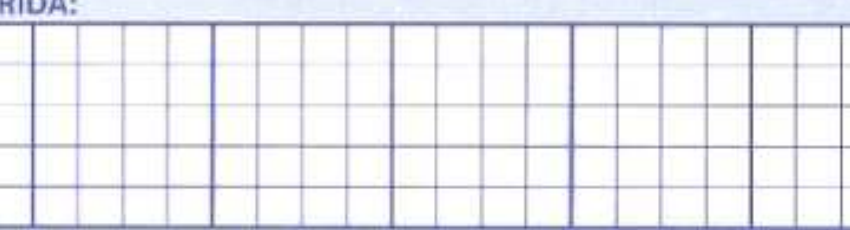

(2)




\section{RESULTADOS.}

El estudio y seguimiento mensual de las upp aparecidas en nuestra UCI durante los 22 meses de control exhaustivo se recogen en el siguiente esquema (valores expresados en porcentajes, prevalencia e incidencia y en valores absolutos la frecuencia) (TABLA 1).

\begin{tabular}{|l|c|c|c|}
\hline \multicolumn{1}{|c|}{2009} & Prevalencia & Incidencia & $\begin{array}{c}\text { Frecuencia } \\
\text { Absoluta }\end{array}$ \\
\hline Abril & 29 & 27 & 17 \\
\hline Mayo & 24 & 25 & 8 \\
\hline Junio & 25 & 17 & 18 \\
\hline Julio & 22 & 11 & 7 \\
\hline Agosto & 21,7 & 13,04 & 15 \\
\hline Septiembre & 22,2 & 14,8 & 7 \\
\hline Octubre & 22,2 & 17,1 & 12 \\
\hline Noviembre & 23,6 & 14,4 & 10 \\
\hline Diciembre & 15,29 & 9,4 & 6 \\
\hline \multicolumn{1}{|c|}{2010} & & & \\
\hline Enero & 15,71 & 10 & 5 \\
\hline Febrero & 16,8 & 14,2 & 8 \\
\hline Marzo & 17,8 & 10,7 & 9 \\
\hline Abril & 15,15 & 4,5 & 4 \\
\hline Mayo & 11,39 & 6,32 & 5 \\
\hline Junio & 12,98 & 9,09 & 7 \\
\hline Julio & 14,28 & 9,5 & 5 \\
\hline Agosto & 12,19 & 7,3 & 5 \\
\hline Septiembre & 13,88 & 9,7 & 8 \\
\hline Octubre & 14,86 & 9,45 & 7 \\
\hline Noviembre & 13,63 & 9,09 & 6 \\
\hline Diciembre & 14,63 & 9,75 & 8 \\
\hline \multicolumn{1}{|c|}{2011} & & & 9 \\
\hline Enero & 12,64 & 10,34 & \\
\hline
\end{tabular}

Tabla 1: tasas periodicidad mensual.

El rango para los valores de las tasas de Prevalencia fue en éstos 22 meses de: 29 - 11,39.

El rango para los valores para las tasas de Incidencia fue de $27-4,5$. Con un comienzo de inflexión, con bajada significativa de las tasas de prevalencia a los ocho meses de introducir las medidas estandarizadas de prevención y mejora asistencial. La inflexión para los datos de incidencia 
comenzó al cuarto mes de comienzo del estudio (Gráfico 1).

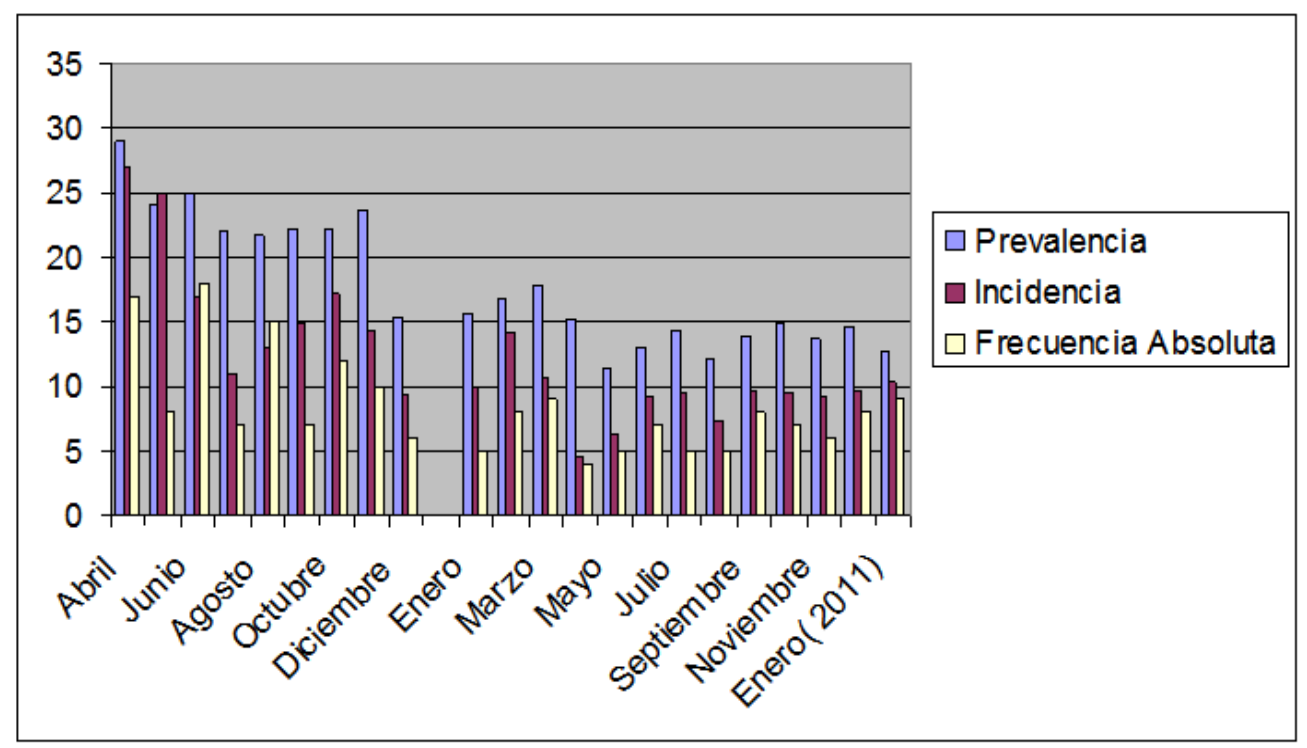

Gráfico 1: evolución de tasas (abril-diciembre 2009, enero-diciembre 2010, enero 2011)

Se hizo una comparativa con los datos de tasas de prevalencia y de incidencia recogidos en nuestra UCI, previos al comienzo de nuestro seguimiento (TABLA 2).

\begin{tabular}{|l|c|c|c|c|}
\hline $\begin{array}{c}\text { Tasas medias } \\
\text { (porcentajes) }\end{array}$ & $\begin{array}{c}\text { Datos pre- } \\
\text { estudio }\end{array}$ & $\begin{array}{c}\text { Datos estudio } \\
\mathbf{( 2 0 0 9 )}\end{array}$ & $\begin{array}{c}\text { Datos estudio } \\
\mathbf{( 2 0 1 0 )}\end{array}$ & $\begin{array}{c}\text { Datos estudio } \\
\text { (globales) }\end{array}$ \\
\hline Prevalencia & 45,98 & 20,22 & 14,30 & 17,76 \\
\hline Incidencia & 45,18 & 16,52 & 9,22 & 12,21 \\
\hline
\end{tabular}

Tabla 2: comparativa de tasas.

\section{CONCLUSIONES.}

La concienciación y la unificación de criterios preventivos y de tratamiento del equipo de Enfermería en la disminución de las tasas de úlceras por presión en nuestra unidad de cuidados intensivos, resultó ser decisiva en la bajada significativa de las mismas y en la consecución de un aumento de la calidad asistencial real percibida por todo el equipo asistencial.

La disminución significativa de las tasas de prevalencia e incidencia de upp constituyó un logro de todo el equipo asistencial de nuestra UCI y 
demostró la eficacia de las medidas de prevención y tratamiento introducidas.

En la actualidad, el equipo de seguimiento y control de las upp continúa en su labor diaria de vigilancia y asesoramiento y establece reuniones periódicas del equipo para discutir problemas, dificultades o dudas que van surgiendo y que son trasmitidas por el equipo asistencial a nuestro grupo de estudio.

\section{AGRADECIMIENTOS.}

Damos nuestras más sinceras gracias a todo el equipo asistencial de la UCI-1 del hospital universitario central de Asturias por su desinteresada colaboración e interés en el buen desarrollo del proyecto de mejora asistencial.

\section{BIBLIOGRAFÍA.}

O Soldevilla JJ, Torra JE. Atención Integral de las Heridas Crónicas. GNEAUPP - SPA. Madrid, 2004.

o García FP, Pancorbo PL, Verdú J, Soldevilla JJ. Rodríguez M, Gago M, Martínez F. Rueda López, J.: Eficacia de los productos para el tratamiento de las úlceras por presión: Una revisión sistemática con metaanálisis GEROKOMOS 2007; 18 : 36-51

o Eaton-Bancroft I. Abordaje multidisciplinario en el tratamiento de las heridas. Nursing 2006; 24:33-35

o Aizpitarte E, García de Galdiano A, Zugazagoitia N, Margall MA, Asiain MC. Úlceras por presión en cuidados intensivos: valoración del riesgo y medidas de prevención. Enferm Intensiva. 2005; $16: 153-63$

o García FP, Pancorbo PL, Torrá JE, Blasco C. Escalas de valoración de riesgo de úlceras por presión. En: Atención Integral de las Heridas Crónicas. GNEAUPP - SPA. Madrid, 2004: 209-216

o Capillas R, Cabré V, Gil AM, Gaitano A, Torra JE. Comparación de la efectividad y coste de la cura en ambiente húmedo frente a la cura tradicional. Ensayo clínico en pacientes de atención primaria con úlceras vasculares y por presión. Rev ROL Enferm 2000; 23: 17-24. 
O Soldevilla JJ, Torra JE, Verdú J, Martínez F, López P, Rueda J, Mayán JM. 20 Estudio Nacional de Prevalencia de Úlceras por Presión en España, 2005. Epidemiología y variables definitorias de las lesiones y pacientes. Gerokomos 2006; 17:154-72.

O Torra i Bou JE et al. $1^{0}$ Estudio nacional de prevalencia y tendencias de prevención de Upp en España. (2001). Gerokomos (2003); 14(1):37-47.

O GNEAUPP. Directrices generales sobre prevención de úlceras por presión. Documentos del GNEAUPP. (Actualizado en 2002; citado el 28 noviembre de 2003).

O Arana Fajardo M; Figueras Bosch MJ. Correlación entre dos escalas de valoración del riesgo de ulceras por presión en pacientes de UCI [Internet]. XXXI Congreso Nacional de la SEEIUC, La Coruña; $2005 ;$ p.48.

- Arcos Díaz-Fuentes M, Barbero López M, Díaz Pizarro JM, García García J, Jiménez Jiménez MC. Protocolo de Actuación en Úlceras por Presión [Internet]. Excelm Enferm 2006.

o Carlos J. Bermejo Caja. Milagros Beamud Lagos. Marisa de la Puerta Calatayud. M. Encarnación Ayuso Gil. Susana Martín Iglesias. M.Carmen Martín-Cocinas Fernández. Fiabilidad interobservadores de dos escalas de detección del riesgo de formación de úlceras por presión en enfermos de 65 o más años. Enferm Clín. Noviembre 1998;8:242-247

o Boletín Terapéutico Andaluz. Tratamiento local de las úlceras por presión.Revisión: Escuela Andaluza de Salud Publica.Bol Ter Andal 2000.

O Bouza Álvarez C, Sanz de León O M, Amate Blanco J M. Efectividad de los Apósitos Especiales en el Tratamiento de las Úlceras por Presión y Vasculares. [Internet]. Agencia de Evaluación de Tecnologías Sanitarias (AETS); 2001 (Informe de Evaluación de Tecnologías Sanitarias No.28).

o Centro para la Difusión de la Investigación (RDC) Tratamiento de las úlceras por presión. Ciudad de Iowa. Centro de Investigación de 
las Intervenciones Gerontológicas de enfermería de la Universidad de Iowa/1997. Revisado en agosto 2002.

o Coria Abel J. Protocolo de prevención y tratamiento de las Úlceras por Presión. [Internet]. Lugo: Hospital da Costa; 2002.

o De Haro Marín S., Navarro Arnedo JM., Orginler Uranga P.E. Enfermería ante las úlceras por presión. Gerokomos 2001; 12: 3943

o Díaz González E. Estudios de enfermería en todos los grados de las úlceras por presión. En: $V$ premio a la investigación en enfermería. Madrid: Lab. Knoll; 1995.p.1-139

o Directrices para el tratamiento de las úlceras por presión. European Pressure Ulcer Advisory Panel (EPUAP): Grupo Europeo de Úlceras por Presión [Internet]. GNEAUPP; 1998 [acceso 2/7/2007].

o Domínguez Rodríguez MC, Trujillo González JM González Méndez T. Úlceras por presión; ...亡 Y ahora que apósito utilizo?[Revista en nternet]. Nure Investigación No 22, mayo-junio 2006.

o Directrices para el tratamiento de las úlceras por presión. European Pressure Ulcer Advisory Panel (EPUAP). [Internet]. 1998.

o Estrategias activas en la prevención de las úlceras por presión. Rev Rol Enferm 2000; 23:314-319. 